\title{
EVENTR - DESIGN OF AN EVENT MANAGEMENT MOBILE APPLICATION USING ONTOLOGY
}

\author{
RAJESH KANNA B ${ }^{1}$, VISWANATHAN V ${ }^{1 *}$, GEORGE EBBINASON AJ ${ }^{2}$
}

${ }^{1}$ School of Computing Science \& Engineering, VIT University, Chennai, Tamil Nadu, India. ${ }^{2}$ Philips India Limited, Bengaluru, Karnataka, India. Email: viswanthan.v@vit.ac.in

Received: 23 January 2017, Revised and Accepted: 03 March 2017

\begin{abstract}
An event is a special occasion that occurs when few or more people gather together to fulfill their personal, cultural or organizational objectives. With many events happening every day, few handy tools are available to help an event organizer to initiate, track and propagate an event. Often important data such as contacts, mails are saved by the organizer in some apps like Excel, calendar, mobile contacts, and mailbox, but not easily retrieved when needed for a follow-up event. Some of the information belongs to common events are not been grouped categorically, so it could not be suitable to relate the similar actions with respect to events which are associated to each other. Follow-up events usually use almost similar data from the previous season, but every time the organizer ends up spending more time searching for the previous records and sometimes fail to retrieve. Event based apps are available in the mobile environment very few in number, and especially such follow-up concentrated event tracker application is not available. Hence, Eventr is designed to fulfill this purpose. Eventr will solve this issue by initiating event, automating simple tasks, record critical data till the event day, this information are stored in the form of Eventr ontology. Since it is ontology based event representation, it would make possible to identify the intermediate tasks of a particular event and to retrieve the tasks associated with the event to the event organizer for the next season.
\end{abstract}

Keywords: Event management, Semantic web, Ontology, Mobile apps.

(C) 2017 The Authors. Published by Innovare Academic Sciences Pvt Ltd. This is an open access article under the CC BY license (http://creativecommons. org/licenses/by/4. 0/) DOI: http://dx.doi.org/10.22159/ajpcr.2017.v10s1.19574

\section{INTRODUCTION}

Event organization/management [1] is an important activity performed throughout a season where season means a single occurrence of a repeating event. Each time the data generated for organizing an event is huge and important. It has the potential to limit organizing overhead for a follow-up event yet actions like mail, call a VIP or a feedback template are not properly noted down or recorded for future use. A search for a solution will yield to an upcoming mobile application called Eventr. A simple time-line based application meant to change the way event organizers initiate, conduct and propagate an event. Applications like event manager lite from key flow are already available, but their focus is not on reusing already collected and available data.

Existing work [2,3] demonstrates how limited information can be used to generate common sense information using machine learning as most tasks are very basic. Instead of using machine learning, we provide the interface needed for the user to log in basic event details and later on after the event is over if the organizer wants to reuse the event details, the user just clicks reinitiate event option which reduces drastically the amount of data needed to be recollected. This ensures that the basic tasks are handled easily by just copying existing samples.

On the other hand, semantic web is an extension of this Web in which the given information is well-defined, better enabling computers and people to work in cooperation $[4,5]$. In the semantic web, ontologies are used to semantically annotate the current information on the Web. Given these ontologies and semantic annotations of data called semantic metadata with respect to them, machines will thus be able to efficiently and in a more automated manner, interpret associated information of the data on the Web [5]. There are a variety of semantic representation to represent the ontology including resource description framework (RDF) and web ontology language. Rule layer is one of the layers in the semantic webcake [5] and it can be created in the form of first order logic. Users can infer the new knowledge from the existing knowledge with the help of rule. SPARQL [6] is a query language for databases which is used to retrieve and manipulate data stored in RDF. With the help of the SPARQL, user can access the ontology and retrieve the relevant information.
Hence, there is a lot of demand for a new system which exploits the power of ontology for the daily activity related event management. This makes Eventr is an ideal choice for event organizers. The importance of creating a workflow toward an event day is realized using Eventr.

\section{MOTIVATION}

The main focus of this research work is derived from affinity analysis conducted through generative research. Event organization, which is a part of group activities requires a considerable amount of efforts to finish successfully. Providing a viable solution to simplify this process is highly looked on. Through generative research, we were able to successfully extract bottom-up information directly from daily users which reveal significant details about what kind of an app would benefit user community.

\section{Research question and methods}

The generative research started with the selection of a field of interest. Author was interested about how groups evolve and how efficiently can a group host an event. Several factors such as interests and members affects how an event happens, and the primary goal was to demystify facts and find a way to help groups to efficiently host an event and hence decided to conduct my study on event organization/management.

The research questions framed helped me to identify potential issues and places of improvement. The questions are as shown below,

1. How participants form an idea for an event? What tools and procedures they use to conduct an event successfully?

2. How do members of the team effectively communicate with each other and stay in sync throughout the event.

3. How members find new people of similar thought to propagate their idea?

4. What are the difficulties in maintaining a plan for the entire event with proper documentation and reports? What present tools are used to simplify them?

5. How last data impacts present repetitive event and what are the challenges faced in follow-up events?

6. What motivates members to work toward a common result? How data shared by each other was crucial to the success of the event? 
All the questions asked in a semi-structured interview or contextual inquiries were based on the above motives. The methods chosen were based on convenience and need.

For this research, basically three methods were chosen. They are,

1. Semi-structured interview.

2. Observation.

3. Contextual inquiry.

\section{Semi-structured interview}

Interview comprised about 50 random semi-structured questions that invoked participants to answer specific to the research needs. 4 members were recruited for the interview out of which three were interviewed through cell phone and one directly through an evening session. Sessions with the participants lasted for about 45-90 minutes. The questions and answers were recorded on a laptop and later replayed to register the important quotes needed for research. Interview was over within 1 day sparing more time for observations and contextual inquiry. Questions were focused on observing what people were doing in the last.

\section{Observations}

In this approach, event members behavior was observed inside and outside group, their ability to conduct an event and the tools they were using to empower event organization. Added to this, around 75 participants were surveyed on how they function in a group and how the group affected their daily life. Although the survey was well conducted with a good questionnaire, important points were registered on what was observed directly which is highlighted as quotes in the notes created.

\section{Contextual inquiry}

Contextual inquiry was conducted along the same time-lines as the interview. Here, the same 4 participants were allowed to talk about a situation and "speak as you think" approach helped to understand how people would behave in certain situations. Necessary questions were asked to prevent participants from deviating and their answers were recorded on laptop for later playback. Above mentioned were the techniques used and did not prefer diary logging as many participants were reluctant to provide in time records about what they were doing and thinking. Initially faced problems with finding the right recruits but later on had the interview and inquiry done with interested people.

\section{Affinity analysis}

The affinity analysis was conducted in the end after gathering all the necessary data. The data, i.e., the quotes that were recorded from participant's voice was each written on single stick notes, and around

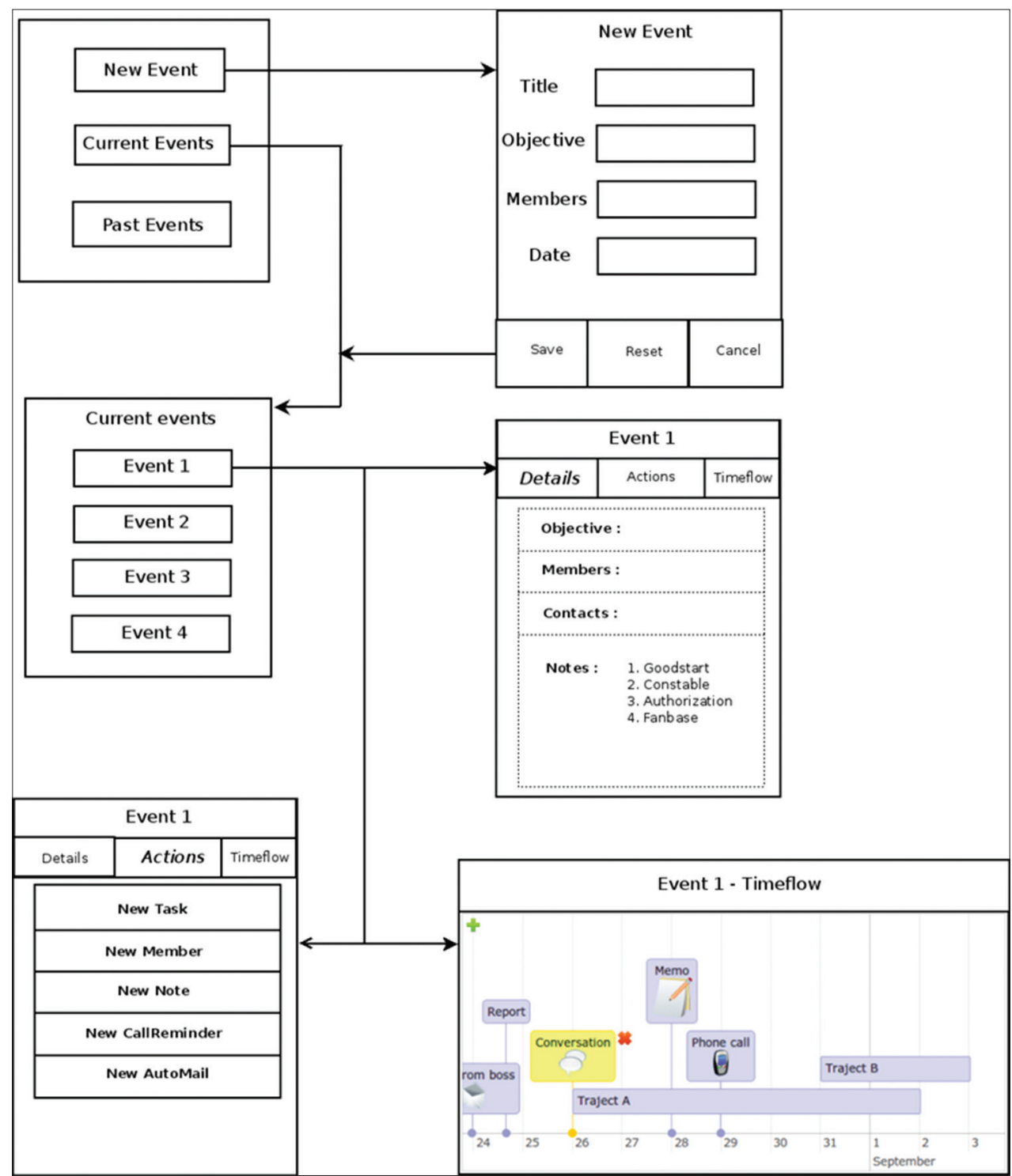

Fig. 1: Eventr prototype 


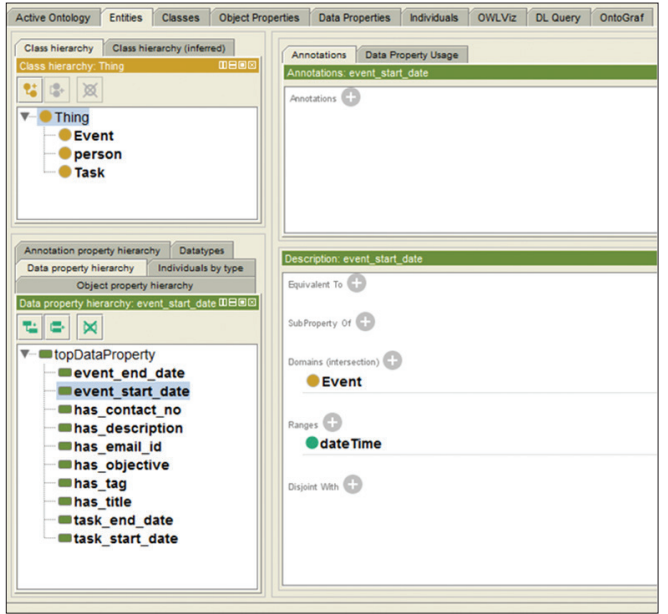

Fig. 2: Structure of Eventr ontology with classes and data properties

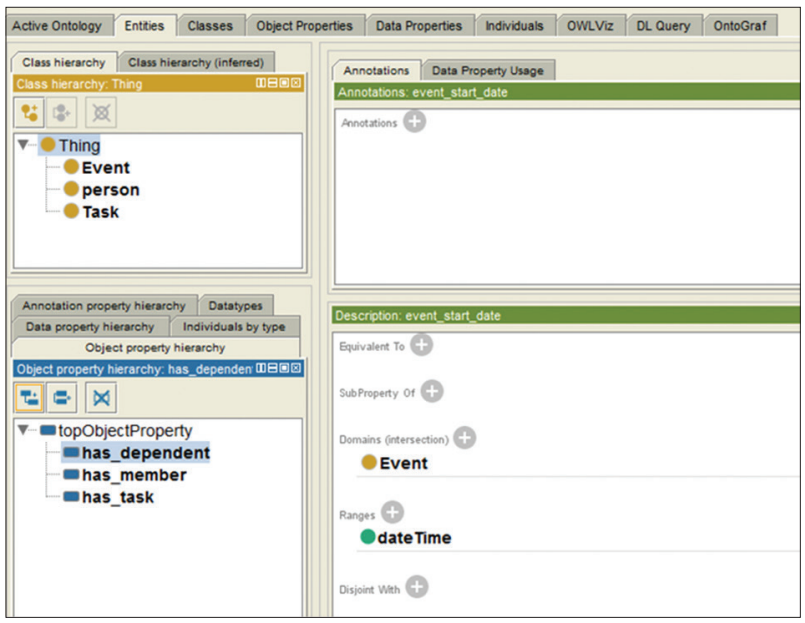

Fig. 3: Structure of Eventr ontology with classes and object properties

80 notes were prepared. We initially, collected the all the tasks related to the event and categorized those tasks based on its behaviour and characteristics. Later made detailed analysis about what each note was talking based on which the aggregation of notes were done on a wall. Some of the themes recorded were:

- Effective communication comes from motivated people. Based on quote "there is a courier boy in our team who was the master mind ... Without him, there would have been no internal communications."

- Formation of core team and importance of assigning work specifically. Based on quote "first a brief mail to everyone about what we are going to do and pick some responsible members from our team to conduct the event."

- Proper administrative work is required. Based on quote "after the plan is confirmed we started a thread in a forum where there will be others also. We put the details there and allowed people to see the cost, plan and asked them to give confirmation if they were interested. We kept it open for 3 days."

- Crisis planning is required to handle unexpected situations. Based on quote "we did not have the first aid kit ready. This was a problem which we realized only in the last day."

- Important things when the event is happening. Based on quote "one important thing $i$ learnt was never leave any gaps in an event. Must be a flow till the end."

- Event recorder is required to simplify organizing a follow-up event: "I don't have a method to save all the contacts, notes, and mails during an event for later retrieval."

It can be seen that one of the participants is emphasizing about tracking an event and another about building a team. One other participant talks about data for later retrieval as the last point. These were the main motivating ideas. Mails and phone call were chosen as the primary means of communication for the application through motivation from effective communication channel theme.

\section{DESIGN OF EVENTR}

Out of all the themes that occurred and discussing in the motivation section, event initiator, tracker, and propagating system was chosen as the main design idea and collectively called as Eventr. The details and designs strategies of the system will be discussed in this session, Fig. 1 shows the prototype of Eventr and below "use case" describes its actions involved in this framework.

\section{Use case}

The key use case for Eventr is as follows,

1. A person decides to conduct an event and creates a new event through the mobile interface.

2. Starts with giving details about the event, members, and the date of the event.

3. User will be able to create self-assigned tasks or tasks for members.

4. Important notes can be saved when and where needed. Locations are recorded along with the notes to let the organizer know where the note was recorded.

5. Organizer will be able to track the tasks through the time-line.

6. Organizer can initiate an automail or autocall task where the person can schedule mail to be sent to multiple people at a particular point in time or schedule a call to remind the organizer to call later.

7. Contacts and mail id's are recorded through these tasks for later retrieval when a similar event is re-initiated.

8. Organizer will be able to re-initiate event or just look at the history to find some needed data.

To store all the actions available in the "use case" Eventr ontology is created and Fig. 2, shows the structure of Eventr ontology with classes and data properties. In Fig. 3, structure of event ontology with classes and object properties has been illustrated.

The design of the application will be similar to what is shown in Fig. 4, and some of the tools needed would be GIMP, Eclipse, Dia and Kompozer. Since all of them are open source tools acquiring them will not be a problem. The application might depend on Google GWT for the time-line to be created which means browser support will be needed.

Once the user enters information about the events, then this information is added into ontology as an instance. This information is provided to the new user to know about the particular event and various sub tasks belongs to this event, event manager, etc. Since the representation of the ontology is in the form of graph, it is possible to identify the association [7] between one task or event and another task or event along with intermediate tasks or events. Hence, the people can understand the prerequisite or priority of each event or task.

For example sequence of marriage reception is as follows:

Bride and groom arrive - formal introduction - receiving line - toast to the bride and groom- invocation - meal service - traditional first dance - cake cutting - father/daughter dance and bouquet toss and garter toss and Fig. 5 shows the overview of the proposed system.

\section{CONCLUSION}

The logical design of the Eventr empowered with the event ontology has been descried in this work. And this design will solve the group communication issues by initiating event, automating simple tasks, 


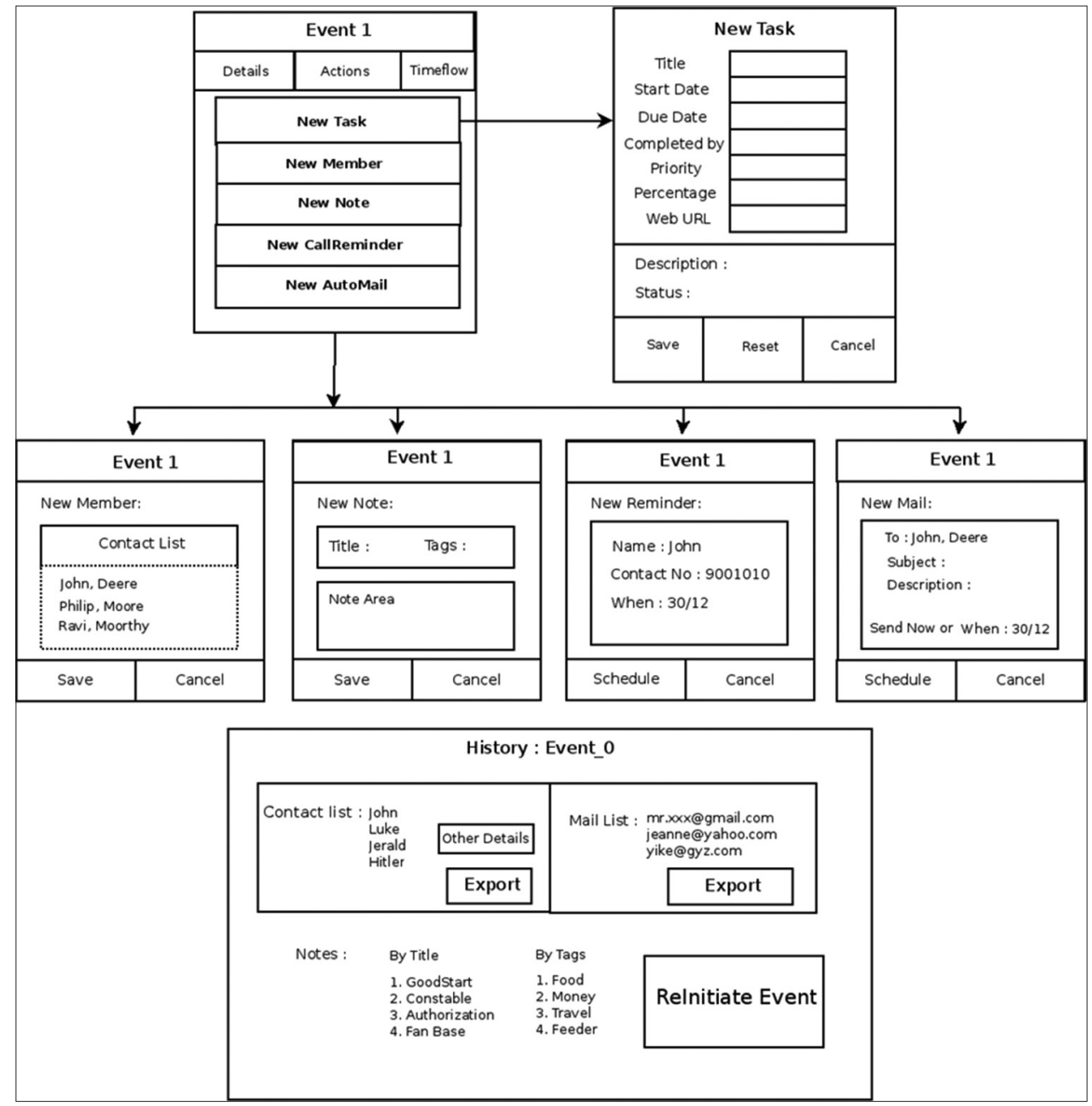

Fig. 4: Tasks and history sections

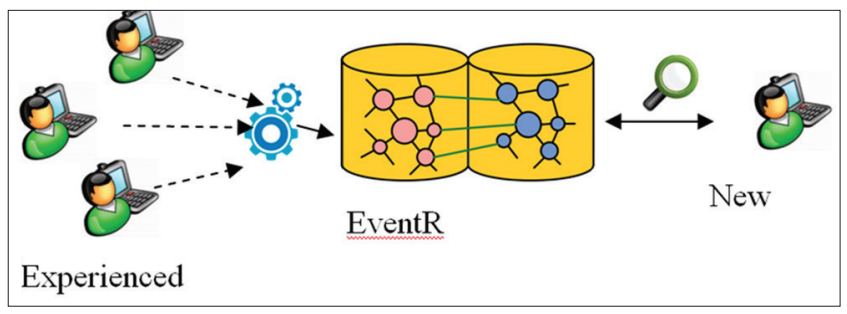

Fig. 5: Overview of Eventr system

record critical data till the event day. Moreover, the proposed ontologybased design would make possible to identify the intermediate tasks of a particular event and to retrieve the tasks associated with the event to the event organizer for the next season.

\section{REFERENCES}

1. Shone A, Parry B. Successful Event Management: A Practical Handbook. London: Thomson Learning; 2004.

2. Smith DA, Lieberman H. Recognizing and using Goals in Event Management. Boston, MA, USA: CHI; 2009.

3. Taleb T, Ksentini A, Chen M, Jantti R. Coping with emerging mobile social media applications through dynamic service function chaining. IEEE Trans Wirel Commun 2016;15(4):2859-71.

4. Lee TB, Hendler J, Lassila O. The semantic web. Sci Am 2001;284(5):34-3.

5. Shadbolt NR, Lee TB, Hall W. The semantic web revisited. IEEE Intell Syst 2006;21(3):96-101.

6. Prud'Hommeaux E, Seaborne A. SPARQL Query Language for RDF. W3C Working Draft; 2006.

7. Viswanathan V, Krishnamurthi I. Finding relevant semantic association paths using semantic ant colony optimization algorithm. Soft Comput 2015;19(1):251-60. 